\title{
Near-infrared intraoperative imaging during resection of an anterior mediastinal soft tissue sarcoma
}

\author{
JARROD D. PREDINA ${ }^{1,2}$, ANDREW D. NEWTON ${ }^{1,3}$, CHARUHAS DESPHANDE $^{1,4}$ and SUNIL SINGHAL SHA $^{1,2}$ \\ ${ }^{1}$ Center for Precision Surgery, ${ }^{2}$ Division of Thoracic Surgery, Department of Surgery, \\ ${ }^{3}$ Division of Hematology and Oncology, Department of Medicine, and ${ }^{4}$ Department of Pathology and Laboratory Medicine, \\ Perelman School of Medicine at The University of Pennsylvania, Philadelphia, PA 19104, USA
}

Received September 6, 2017; Accepted November 6, 2017

DOI: $10.3892 / \mathrm{mco} .2017 .1491$

\begin{abstract}
Sarcomas are rare malignancies that are generally treated with multimodal therapy protocols incorporating complete local resection, chemotherapy and radiation. Unfortunately, even with this aggressive approach, local recurrences are common. Near-infrared intraoperative imaging is a novel technology that provides real-time visual feedback that can improve identification of disease during resection. The presented study describes utilization of a near-infrared agent (indocyanine green) during resection of an anterior mediastinal sarcoma. Real-time fluorescent feedback provided visual information that helped the surgeon during tumor localization, margin assessment and dissection from mediastinal structures. This rapidly evolving technology may prove useful in patients with primary sarcomas arising from other locations or with other mediastinal neoplasms.
\end{abstract}

\section{Introduction}

Primary mediastinal sarcomas are rare (1). Similar to other primary sarcomas, achieving long-term survival often requires multimodal therapy including complete local resection, chemotherapy, and radiation (2). Accomplishing complete resection of anterior mediastinal sarcomas poses challenges given proximity to critical mediastinal structures such as the phrenic nerve, heart, and great vessels. Accurate tumor identification and margin assessment during mediastinal dissection is further complicated when occurring after neoadjvant chemotherapy, an approach commonly implemented for sarcomas.

Our group has previously reported successful utilization of near-infrared (NIR) fluorescent, real-time intraoperative

Correspondence to: Dr Sunil Singhal, Center for Precision Surgery, Perelman School of Medicine at The University of Pennsylvania, 6 White Building, 3400 Spruce Street, Philadelphia, PA 19104, USA

E-mail: sunil.singhal@uphs.upenn.edu

Key words: soft tissue sarcoma, neoadjuvant chemotherapy, surgery, intraoperative imaging, thymic carcinosarcoma imaging to improve pulmonary metastasectomy (3). In this report, we detail successful utilization of this approach to enhance accurate identification of malignancy during resection of a thymic carcinosarcoma in a patient that previously underwent neoadjuvant chemotherapy. This report highlights how this approach can improve a surgeon's ability to identify disease and safely obtain adequate margins during resection of primary mediastinal sarcomas and, perhaps more broadly, other solid tumors located near critical structures.

\section{Case report}

A 59-year-old male was seen in our multi-disciplinary thoracic oncology institute for management of an incidentally identified 5.7x7.5x8.4 cm anterior mediastinal mass (Fig. 1A). This mass was abutting the left pulmonary artery without obvious vascular invasion. Metastatic work-up revealed no suspicious metastases. A transthoracic needle biopsy was suspicious for a thymic carcinosarcoma with rhabdomyosarcomatous elements. Six rounds of neoadjuvant chemotherapy with Adriamycin, Ifosfosfamide and Vinicristine. After completing neoadjuvant treatment, the patient was consented for resection via left thoracosternotomy with NIR intraoperative imaging.

Twenty-four hours before resection, intravenous indocyanine-green (ICG) was delivered (5 mg/kg). During resection, the tumor displayed high levels of fluorescence (tumor-to-background signal ratio of 3.6) (Fig. 1B and C). Real-time fluorescent feedback aided the surgeon when during dissection from critical mediastinal structures including the phrenic nerve, pulmonary artery and aorta. After resection, negligible residual fluorescence (tumor-to-background signal ratio of 1.2) was appreciated within the operating field which increased the surgeon's confidence of complete resection (Fig. 1D).

On backtable evaluation, tumor samples were obtained from 4 quadrants (Fig. 1C and E) and fluorescence was evaluated using a macroscopic NIR fluorescence system (Visionsense ${ }^{\circledR}$, Philadelphia, PA) (Fig. 1F). Specimen were then evaluated using a microscopic NIR fluorescent imaging system (LiCor Odyssey, Lincoln, NE) and by H\&E staining (Fig. $1 \mathrm{G}$ and $\mathrm{H}$ ). Margins were clear thus confirming findings of intraoperative fluorescent imaging. Of note, utilization of intraoperative imaging added $11 \mathrm{~min}$ to the case duration. 


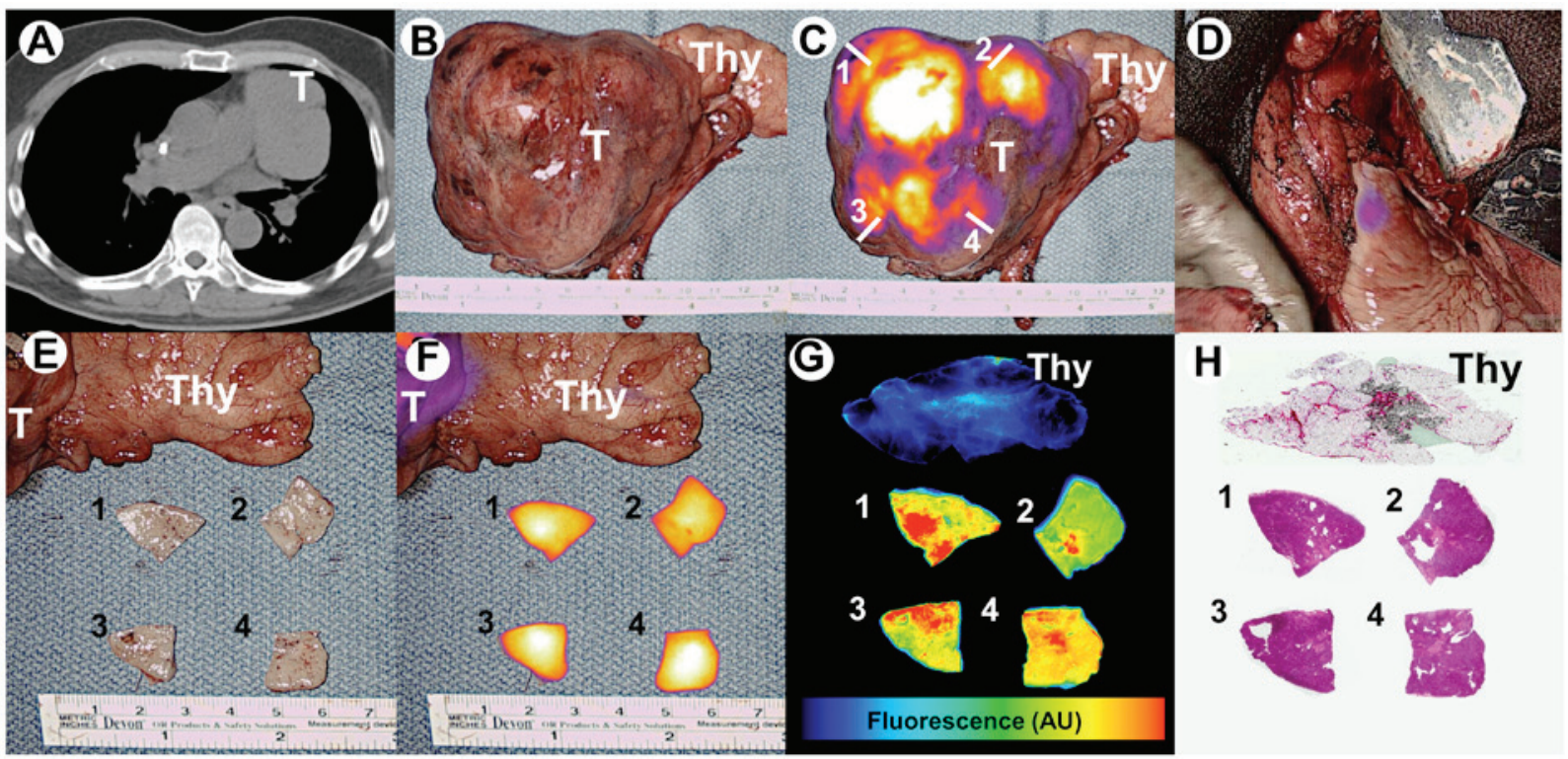

Figure 1. Preoperative and intraoperative NIR imaging of thymic carcinosarcoma: (A) preoperative CT demonstrating $4.6 \times 7.6 \mathrm{~cm}$ anterior mediastinal mass, (B) white-light imaging of en bloc resection involving 10.0x9.0x3.7 cm tumor with benign thymic tissue, (C) fluorescent imaging of en bloc resection with denotation of breadloaf sections (labeled 1-4), (D) post-resection wound bed with negligible fluorescence, (E) White-light views of breadloaf sections and benign thymus, (F) macroscopic fluorescent imaging of breadloaf sections and thymus, (G) microscopic fluorescent imaging of specimens, (H) H\&E microscopy of specimens. T-tumor; Thy-thymus; 1-4 breadloaf sectioning of tumor; AU-arbitrary units.

Histopathologic analysis revealed a biphasic tumor, composed of spindle and rhabdoid morphology cells along with epithelioid cells in other regions. Upon immunohistochemical analysis, spindle and rhabdoid cells stained positive for desmin, myogenin, smooth muscle actin, HHF-35, and INI-1; patchy weak positivity for S100 and MelanA was also noted. Epithelioid cells were noted to be positive for AE1/3, CAM5.2, CK5/6, and p40; weak positivity for PAX8 was also found. Overall, this morphology and immunohistochemical staining pattern was consistent with thymic carcinosarcoma with rhabdomyosarcomatous elements.

The subject recovered uneventfully without drug toxicity and remains disease free after 1 year of follow-up.

\section{Discussion}

In this report, we provide preliminary evidence suggesting safety and feasibility of NIR intraoperative imaging with ICG for anterior mediastinal sarcomas after neoadjuvant therapy. Real-time fluorescent feedback provides additional visual information that can help the surgeon during tumor localization, margin assessment and dissection from nearby vital structures.

ICG has traditionally been utilized by clinicians to assess perfusion and determine vascularity (4). Our group has recently described ICG as a tumor mapping agent (3). For tumor mapping, ICG is delivered at a dose of $5 \mathrm{mg} / \mathrm{kg}$ with imaging occurring $24 \mathrm{~h}$ later. Under these dosing parameters, ICG functions by exploiting abnormally leaky capillaries and increased pressure gradients (the Enhanced Permeability and Retention Effect) which are found in most solid malignancies, including sarcomas (5). In this report, we found optical properties of ICG to be excellent for intrathoracic imaging. One reconcilable drawback, however, was persistent low levels of background fluorescence from the aorta, which is likely due to low levels of circulating albumin bound ICG. Nevertheless, accurate tumor identification was feasible given significantly higher levels of tumor-specific signal.

Although additional studies are needed, these initial findings remain encouraging. First, delivery of this dosage of ICG was well tolerated, which is consistent with our previous experiences with ICG (3). Second, we found that the addition of intraoperative imaging was efficient and addended only several minutes to the case duration. Third, reliable real-time fluorescent feedback ( $100 \%$ accuracy) assisted the surgeon during dissection from critical mediastinal structures and suggested complete resection. Lastly, we found high levels of fluorescence after neoadjuvant chemotherapy, an approach that is applied to a variety of solid tumors, including sarcomas. Further investigation of this approach in patients with primary sarcomas and other mediastinal neoplasms, such as ectopic parathyroid adenomas and thymoma, is ongoing (NCT02280954). In the future, this technology may be a reliable tool to enhance a surgeon's ability to perform a variety of oncologic procedures including tumor localization, margin assessment and intraoperative staging.

\section{Acknowledgements}

JDP was supported by a grant from the American Philosophical Society, the NIH (F32 CA210409) and the Association for Academic Surgery Research Grant. SS was supported by the NIH (R01 CA193556).

\section{References}

1. Gladish GW, Sabloff BM, Munden RF, Truong MT, Erasmus JJ and Chasen MH: Primary thoracic sarcomas. Radiographics 22: 621-637, 2002. 
2. Gutowski CJ, Basu-Mallick A and Abraham JA: Management of bone sarcoma. Surg Clin North Am 96: 1077-1106, 2016.

3. Keating J, Newton A, Venegas O, Nims S, Zeh R, Predina J, Deshpande C, Kucharczuk J, Nie S, Delikatny EJ and Singhal S: Near-infrared intraoperative molecular imaging can locate metastases to the lung. Ann Thorac Surg 103: 390-398, 2017.

4. Boni L, David G, Mangano A, Dionigi G, Rausei S, Spampatti S, Cassinotti E and Fingerhut A: Clinical applications of indocyanine green (ICG) enhanced fluorescence in laparoscopic surgery. Surg Endosc 29: 2046-2055, 2015.
5. Jiang JX, Keating JJ, Jesus EM, Judy RP, Madajewski B, Venegas O, Okusanya OT and Singhal S: Optimization of the enhanced permeability and retention effect for near-infrared imaging of solid tumors with indocyanine green. Am J Nucl Med Mol Imaging 5: 390-400, 2015. 